Bio - grafía. Escritos sobre la Biología y su Enseñanza. ISSN 2027-1034

Edición Extraordinaria. p.p. 420- 430

Memorias del IX Encuentro Nacional de Experiencias en Enseñanza de la Biología y la

Educación Ambiental. IV Congreso Nacional de Investigación en Enseñanza de la Biología.

\title{
ARTICULACIÓN ENTRE ESTILOS DE APRENDIZAJE Y EL DESARROLLO DE ESTRATEGIAS DE ENSEÑANZA EN PROFESORES EN EJERCICIO DE CIENCIAS DE LA GUAJIRA - COLOMBIA
}

\author{
Carlos Humberto Barreto Tovar ${ }^{1}$ \\ Pedro Ramírez Sánchez ${ }^{2}$ \\ Yulieth Romero Rincón ${ }^{3}$
}

\section{RESUMEN}

Todos los estudiantes presentes en el aula de clase están dotados de importancia e individualidad. En esta investigación 26 estudiantes - profesores de ciencias en ejercicio de un programa de posgrado de La Guajira - Colombia respondieron el Index of Learning Styles Questionnaire, desarrollado por Felder y Soloman (1991). Los resultados fueron agrupados en frecuencias en relación a la categoría predominante y en ellas a su tendencia fuerte, moderada o equilibrada. En ellos se evidenció una mayor tendencia en las dimensiones Visual (61.5\%), Secuencial $(65,4 \%)$ y Activo $(61,5 \%)$.

Para el análisis se utilizó la correlación de Pearson como herramienta estadística, determinándose una correlación positiva entre las tendencias del grupo y la distribución de tendencias por género. A partir de estos valores y bajo el marco de la Enseñanza para la Comprensión (EpC), los docentes responsables de la formación de estudiantes profesores reflexionaron sobre su quehacer pedagógico planteando estrategias de aprendizaje que fueron desarrolladas en el curso del Énfasis en Docencia para el Desarrollo del Pensamiento Científico y Tecnológico de la Maestría en Pedagogía.

PALABRAS CLAVES: Estilos de aprendizajes, estrategias de enseñanza, formación continuada de docentes de ciencias, conocimiento profesional del profesor de ciencias, enseñanza para la comprensión (EpC).

\section{ABSTRACT}

All students present in the classroom are endowed with importance and individuality. In this research 26 students - science teachers in a postgraduate program in La Guajira Colombia answered the Index of Learning Styles Questionnaire, developed by Felder and

\footnotetext{
${ }^{1}$ Universidad de La Sabana. Facultad de Educación. Maestría en Pedagogía. Correo: carlos.barreto2@unisabana.edu.co

${ }^{2}$ Universidad de La Sabana. Facultad de Educación. Maestría en Pedagogía. Correo: pedrorasa@unis abana.edu.co

${ }^{3}$ Universidad de La Sabana. Facultad de Educación. Maestría en Pedagogía. Correo: yuliethrori@unisabana.edu.co
} 
Bio - grafía. Escritos sobre la Biología y su Enseñanza. ISSN 2027-1034

Edición Extraordinaria. p.p. 420- 430

Memorias del IX Encuentro Nacional de Experiencias en Enseñanza de la Biología y la

Educación Ambiental. IV Congreso Nacional de Investigación en Enseñanza de la Biología.

Soloman (1991). The results were grouped in frequencies in relation to the predominant category and in them to their strong, moderate or balanced tendency. In them, a greater tendency was observed in the Visual (61.5\%), Sequential (65.4\%) and Active (61.5\%) dimensions.

For the analysis, Pearson's correlation was used as a statistical tool, determining a positive correlation between the trends of the group and the distribution of tendencies by gender. From these values and under the framework of Teaching for Understanding (EpC), the teachers responsible for the formation of students - teachers reflected on their pedagogical task, proposing strategies of learning that were developed in the course of the Teaching for the Development of Thought Scientific and Technological Department of the Master's Degree in Pedagogy.

KEY WORDS: Learning styles, teaching strategies, continuing education of science teachers, professional knowledge of the science teacher, Teaching for Understanding (EpC).

\section{INTRODUCCIÓN}

Caracterizar la forma en que los profesores en formación posgradual perciben, entienden, transforman y proponen sus dinámicas académicas de enseñanza por un lado y de aprendizaje en otro, ha sido un reto pedagógico continuo en muchos ámbitos académicos nacionales e internacionales, llevando a pensar que todo aquello que se evidencia en el aula formadora, es también una expresión implícita de lo que el estudiante docente es en su aula, como profesor formador. Para Sánchez y Andrade, mencionados por Chiang (Chiang, 2016), se puede lograr un buen efecto en la calidad del aprendizaje si se logran seleccionar estrategias y actividades que son prácticas, y que puedan probarse en el aula de clase en distintas ocasiones, de tal forma de permita quedarse con las que funcionan, eliminar las que no y probar una o dos nuevas.

En el mismo sentido Chiang, al mencionar a Jensen (2004) y Walsh (2011), exalta que la instrucción diferenciada es un medio que involucra muchos estilos de enseñanza con la provisión de múltiples oportunidades para los diversos estilos de aprendizaje; queriendo con ello manifestar que los estudiantes pueden utilizar sus estilos, preferencias e intereses individuales, con el fin de lograr el éxito académico. Sin duda alguna, el estilo de aprendizaje está asociado a como el estudiante percibe, interacciona y responde en un ambiente de aprendizaje, Huizar (2009). Así, es posible estudiar en el ámbito educativo estos estilos de aprendizaje para cada estudiante y a partir de ellos, desarrollar una serie de estrategias definidas por Beltrán (2003) como "operaciones o actividades mentales que facilitan y desarrollan los diversos procesos de aprendizaje" y que adoptan la denominación de herramientas cognitivas útiles a los individuos para la realización de tareas específicas. Estas estrategias pretenden el desarrollo cognitivo y pretenden el aprendizaje o la visibilización de éste. 


\section{Bio - grafía. Escritos sobre la Biología y su Enseñanza. ISSN 2027-1034}

Edición Extraordinaria. p.p. 420- 430

Memorias del IX Encuentro Nacional de Experiencias en Enseñanza de la Biología y la

Educación Ambiental. IV Congreso Nacional de Investigación en Enseñanza de la Biología.

En concordancia con las actividades mentales, las estrategias de enseñanza, las múltiples oportunidades, se establecen para cada alumno, características distintivas que definen los ejes de su proceso de aprendizaje sin que ello implique que los alumnos -profesores en ejercicio- cuenten con un marco de referencia que permita caracterizar el aprendizaje en ellos. Tavares (2007), abre la discusión en torno a un elemento difuso, la relación que se debe dar entre las estrategias de enseñanza desarrolladas por los profesores de posgrado y las tendencias del estilo de aprendizaje que presentan los estudiantes. Encontrar dicha relación, significa identificar cuáles son las estrategias que presenta un profesor frente a su disciplina, cuáles son las actividades que privilegian los aprendizajes asociados con las tendencias en los estilos de aprendizaje, cuáles elementos promueven la integración entre estilos, cuáles acciones le permiten evidenciar un proceso o simplemente cuántos de esos estudiantes poco visibles del aula cobran protagonismo cuando las acciones promotoras del aprendizaje le brindan elementos de discusión.

Esto se lograr en especial cuando Lankard (2003) advierte que los profesores deben examinar su propio sistema de creencias sobre la educación y como ser partícipes de un proceso continuo de diagnóstico con ellos mismos y sus estudiantes, que implique observación, cuestionamiento, retroalimentación evaluativa y la reflexión crítica (Kiernan, 2004). En ello será posible encontrar por lo menos tres grandes reflexiones primero como docentes formadores de docentes a la vez que formamos, revisamos nuestras estructuras de enseñanza, desde la premisa de enseñar bien para aprender bien; en segundo lugar quien está en formación entenderá que sus cuestionamientos se dan en el marco de un desequilibrio de aquello que consideraron bien trabajado, a pesar de que sus estudiantes no alcanzaran éxito en un alto porcentaje; y en tercer lugar, un proceso de diagnóstico y reflexión debe estar soportando el quehacer docente continuamente, de tal suerte que se genere un proceso auto reflexivo y de metacognición de su proceso de enseñanza.

Como objetivo de la investigación se planteó identificar las estrategias de enseñanza en la formación de estudiantes - profesores en ejercicio - del Énfasis de Docencia para el Desarrollo del Pensamiento Científico y Tecnológico, que estén en relación con los estilos de aprendizaje del grupo.

\section{METODOLOGÍA}

El grupo de estudio estuvo integrado por 26 estudiantes - profesores en ejercicio de un programa de posgrado de La Guajira - Colombia, de los cuales 10 son mujeres $(38,5 \%)$ y 16 son hombres $(61,5 \%)$, todos con titulaciones de pregrado en diversos campos de formación relacionados con las Ciencias Naturales y las Ciencias Sociales y que desempeñan sus funciones profesionales en los diferentes grados de educación escolar: primaria, secundaria y media.

La investigación es de carácter cualitativo y se configura como un estudio descriptivo Interpretativo, buscando la modificación de la práctica docente desde la reflexión de las situaciones al interior del aula. Para determinar la tendencia en el estilo de aprendizaje se aplicó la prueba Index of Learning Styles Questionnaire (ILS: Índice de Estilos de 
Bio - grafía. Escritos sobre la Biología y su Enseñanza. ISSN 2027-1034

Edición Extraordinaria. p.p. 420- 430

Memorias del IX Encuentro Nacional de Experiencias en Enseñanza de la Biología y la

Educación Ambiental. IV Congreso Nacional de Investigación en Enseñanza de la Biología.

Aprendizaje), Felder y Soloman (1991). La prueba hace referencia a cuatro dimensiones relacionadas con: 1 . El tipo de información que perciben preferiblemente los estudiantes (sensitivo/intuitivo); 2. El tipo de estímulo en relación a la modalidad sensitiva en que es recibida la información cognitiva (visual/verbal); 3 . La forma de procesar y comprender la información (secuencial/global) y 4. La dimensión relativa con la forma como trabaja con la información (activo/reflexivos). La prueba original proporciona una traducción al español, que fue ajustada para su comprensión en el contexto colombiano validándose con pares académicos y en diferentes escenarios y grupos de edades, permitiendo hacer ajustes en lenguaje y en la intención real de cada pregunta.

En relación al acceso, aplicación y análisis de los resultados, se obtuvo y adaptaron las preguntas, manteniendo el idioma español y se aplicó el test en físico, en igualdad de condiciones de tiempo, espacio, libertad y acompañamiento a todos los participantes de la investigación. Los datos fueron ingresados a la plataforma web de la prueba y desde allí obtener el valor de tendencias en cada una de las dimensiones. Estos valores fueron organizados en hojas de cálculo del programa Excel, por la versatilidad para el análisis, el diseño de gráficas y la aplicación de fómulas o funciones estadísticas como medida de tendencia central o correlaciones. Al obtener, organizar y analizar los datos, se logra elaborar un perfil individual y grupal de estilos de aprendizaje. La correlación de Pearson, se aplicó en relación a los datos generales del grupo en cuanto a la distribución porcentual en las dimensiones de estilos de aprendizaje y la incidencia desde el análisis por género en la muestra.

\section{RESULTADOS Y DISCUSIÓN}

\section{Identificación de estilos de aprendizaje}

La gráfica 1 muestra una distribución porcentual según las tendencias en cada una de las categorías identificadas. Es importante resaltar los valores de $61.5 \%$ de las categorías Activa sobre la reflexiva y de la Visual sobre la verbal; y de $65,4 \%$ de la categoría secuencial sobre la global, para el que los profesores responsables del curso estimaron la planeación de actividades, la inclusión de ejercicios que atendieran dichas tendencias activas, visuales y secuenciales. Además, se identificaron las tendencias predominantes en los grupos de mujeres y hombres. Para las mujeres, la distribución porcentual es semejante en las dimensiones Activo (63,6\%) / Reflexivo (36.4\%) y Visual $(63,6 \%)$ / Verbal (36.4\%). Para el grupo de hombres, la tendencia presenta porcentajes iguales de $60 \%$ en las categorías Activo, sensitivo, Visual y sobresale en valor la tendencia secuencial $(73,3 \%)$ sobre la global. 
Bio - grafía. Escritos sobre la Biología y su Enseñanza. ISSN 2027-1034

Edición Extraordinaria. p.p. 420- 430

Memorias del IX Encuentro Nacional de Experiencias en Enseñanza de la Biología y la

Educación Ambiental. IV Congreso Nacional de Investigación en Enseñanza de la Biología.

Gráfica 1. Tendencias en los estilos de aprendizaje de los estudiantes - docentes.

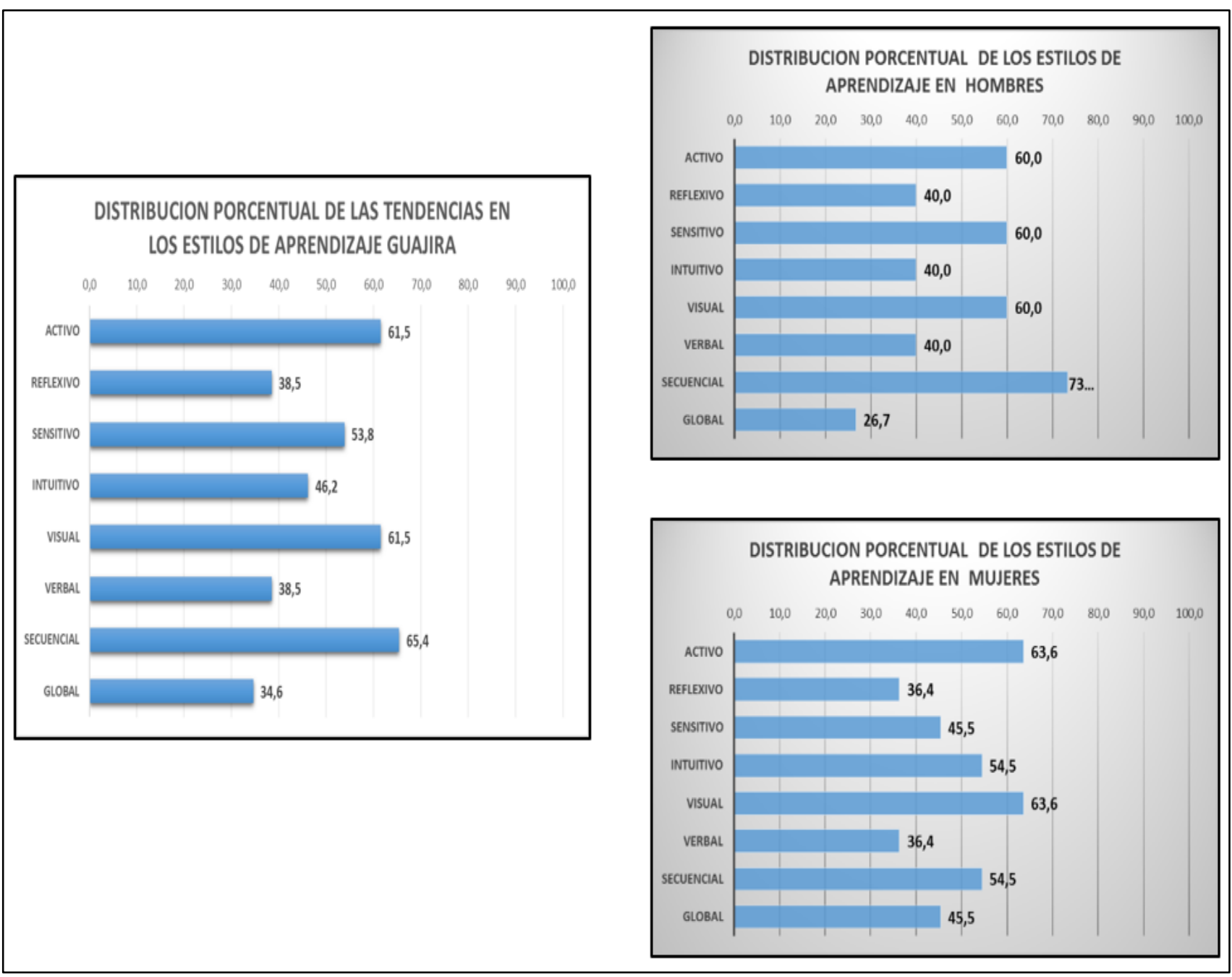

El abordaje, desarrollo y presentación de resultados dejan en evidencia que las tendencias Activa, Visual y Sensitiva de este grupo, facilitan organizar información, socializarla y como lo plantea Castaño (2004), establecer el modo de percibir, interaccionar y responder ante situaciones de aprendizaje no depende del nivel intelectual, sino de las preferencias por percibir y comprender el entorno de forma más tangible o de forma más abstracta y la preferencia por procesar y transformar la información de forma más activa o reflexiva, por lo cual las acciones que se emprenden como estrategias en el grupo deben atender dichas tendencias. 


\section{Bio - grafía. Escritos sobre la Biología y su Enseñanza. ISSN 2027-1034}

\section{Edición Extraordinaria. p.p. 420- 430}

Memorias del IX Encuentro Nacional de Experiencias en Enseñanza de la Biología y la

Educación Ambiental. IV Congreso Nacional de Investigación en Enseñanza de la Biología.

De la misma forma en que se caracteriza al grupo, es posible establecer una caracterización individual que le permite al estudiante, reconocer sus tendencias en el estilo de aprendizaje, de una parte, en forma visual - gráfica como se observa en la parte inferior y de igual manera en forma descriptiva. Al conocer el perfil se solicitó a las personas que establecieran una reflexión, en la búsqueda de interiorizar los elementos dados. En relación directa con el informe individual, se debe generar una socialización de resultados grupales, con intención de permitir que el grupo se identifique con sus pares, establezca nuevos nexos de trabajo, genere dinámicas de agrupación basados en interés de estilos y permitir a la vez entablar un diálogo en función de las nuevas estrategias que se van a implementar dentro del espacio académico de formación, teniendo en cuenta que es un curso diverso desde otros parámetros, además de generar al docente un nuevo elemento motivante como profesor reflexivo, transformador y motivador.

Tabla 1. Tendencias en los estilos de aprendizaje de los estudiantes - docentes en relación a cada categoría.

\begin{tabular}{|c|c|c|c|c|c|c|c|c|}
\hline \multirow{3}{*}{ AGRUPACION GENERAL POR CATEGORIAS. } & 16 & 10 & 14 & 12 & 16 & 10 & 17 & 9 \\
\hline & ACtIVO & REFLEXIVO & SENSITIVO & INTUITIVO & VISUAL & VERBAL & SECUENCIAL & GLOBAL \\
\hline & 61,5 & 38,5 & 53,8 & 46,2 & 61,5 & 38,5 & \begin{tabular}{|l|l}
65,4 \\
\end{tabular} & 34,6 \\
\hline TENDENCIA MODERADA O FUERTE & 9 & 3 & 5 & 2 & 10 & 7 & 8 & 2 \\
\hline PORCENTAJES & 56,3 & 30,0 & 35,7 & 16,7 & 62,5 & 70,0 & 47,1 & 22,2 \\
\hline & ACTIVO M-F & REFLEXIVO & SENSITIVO & INTUITIVO & VISUAL & VERBAL & SECUENCIAL & GLOBAL \\
\hline & 56,3 & 30,0 & 35,7 & 16,7 & 62,5 & 70,0 & \begin{tabular}{|l|l|}
47,1 \\
\end{tabular} & 22,2 \\
\hline TENDENCIA EQUILIBRADA & 7 & 7 & 9 & 10 & 6 & 3 & 9 & 7 \\
\hline $\begin{array}{l}\text { PORCENTAIES } \\
\end{array}$ & 43,8 & 70,0 & 64,3 & 83,3 & 37,5 & 30,0 & 52,9 & 77,8 \\
\hline & ACTIVO-E & REFLEXIVO & SENSITIVO & INTUITIVO & VISUAL & VERBAL & SECUENCIAL & GLOBAL \\
\hline & 43,8 & 70,0 & \begin{tabular}{|l|}
64,3 \\
\end{tabular} & 83,3 & 37,5 & 30,0 & 52,9 & 77,8 \\
\hline
\end{tabular}

La tabla 1 permite establecer la distribución de las dimensiones, en relacion con la tendencia en cada categoria. Aparecen resaltados en color los valores según la siguiente interpretacion: En la dimension Activo / reflexivo, la categoria activa es mayoritaria con un $61,5 \%$, de los cuales el 56,3\% presentan una tendencia Moderada o fuerte, lo que impica criterios de planeacion que atiendan estos aspectos. De igual manera en la dimension Sensitivo /Intuitivo, la categoria sensitiva es mayoritaria con un $53,8 \%$, de los cuales el $64,3 \%$ presentan una tendencia Equilibrada, por tanto el grupo puede ser involucrado desde acciones sensitivas - intuitivas. En relacion a la dimension Visual / Verbal, la categoria predominante es la visual con un $61,5 \%$, de los cuales un $62,5 \%$ son de tendencia moderada o fuerte. Finalmente del $65,4 \%$ de la categoria secuencial sobre la global, un $52,9 \%$ es de tendencia equilibrada, pero es al $47,1 \%$ de tendencia fuerte 0 moderada al que deben generársele acciones incluyentes y de atencion, en especial cuando es posible entender que en todas las categorias una condicion equilibrada le permite a los estudiantes adaptarse a una $u$ otra tendencia desde la estrategias intencionadas. 
Bio - grafía. Escritos sobre la Biología y su Enseñanza. ISSN 2027-1034

Edición Extraordinaria. p.p. 420- 430

Memorias del IX Encuentro Nacional de Experiencias en Enseñanza de la Biología y la

Educación Ambiental. IV Congreso Nacional de Investigación en Enseñanza de la Biología.

\section{Estrategias de enseñanza}

Desde esta identificación, el equipo de profesores desarrolló la propuesta desde la implementación de estrategias enmarcadas en Enseñanza para la comprension (EpC), entre ellas rutinas de pesamiento como GCCE, por las siglas de Generar, Clasificar, Conectar y Elaborar, que visibilizaron el pensamiento en el aula especialmente a estudiantes Intuitivos, quienes identificaronn elementos claves de una o más lecturas simultáneas, las organizaron según criterios propios, los compartieron en la construcción de organizadores gráficos y desde allí mostraron nuevas capacidades para elaborar una sintesis de forma integrada y con apoyo constante del equipo de compañeros. Elaborar un esquema, integrar informacion en organizadores gráficos, desarrollar las ideas de un texto o varios en un mapa mental, está en relacion directa con la forma en que define Cazau (2004) las tendencias visuales y secuenciales. Identificar y llevar al espacio de clase, una caracterización de los estudiantes, desde su estilo de aprendizaje, permite transformaciones integrales en el proceso de Enseñanza y Aprendizaje, necesariamente en la acción pedagógica del docente y en la disposición y percepción desde el estudiante - profesor en ejercicio de posgrado.

Tabla 2. Resumen de algunas estrategias, utilizadas como medio de atención e inclusión en el desde la tendencia en el estilo de aprendizaje.

\begin{tabular}{|c|c|c|c|c|}
\hline $\begin{array}{c}\text { Estrategi } \\
\quad a\end{array}$ & $\begin{array}{c}\text { Nombre de la } \\
\text { estrategia }\end{array}$ & Descripción & $\begin{array}{c}\text { Estilo de } \\
\text { Aprendizaje } \\
\text { que privilegia }\end{array}$ & $\begin{array}{l}\text { Estrategia de } \\
\text { evaluación o tipo de } \\
\text { evaluación que } \\
\text { involucra. }\end{array}$ \\
\hline 1 & $\begin{array}{l}\text { RUTINA DE } \\
\text { PENSAMIENT } \\
\text { O GCCE }\end{array}$ & \begin{tabular}{lr} 
Rutina & para \\
sintetizar & $y$ \\
organizar ideas. \\
Busca describir & y \\
organizar & el \\
conocimiento previo \\
para identificar \\
\multicolumn{2}{l}{ conexiones }
\end{tabular} & $\begin{array}{c}\text { REFLEXIVO - } \\
\text { GLOBAL - } \\
\text { VERBAL }\end{array}$ & 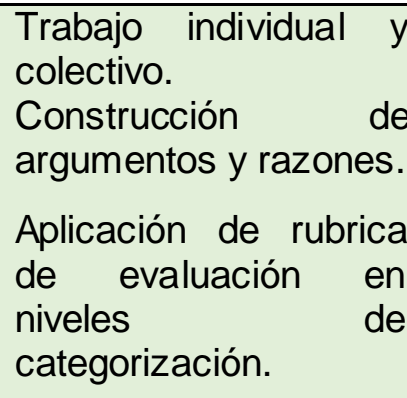 \\
\hline 2 & $\begin{array}{l}\text { GRABACIÓN } \\
\text { DE CLASE }\end{array}$ & $\begin{array}{l}\text { Desde estrategias } \\
\text { de planeación, en } \\
\text { este caso desde } \\
\text { enseñanza para la } \\
\text { comprensión, } \\
\text { ejecuta los } \\
\text { momentos de clase } \\
\text { y los deja en } \\
\text { evidencia a través } \\
\text { de grabación, que }\end{array}$ & $\begin{array}{l}\text { REFLEXIVO - } \\
\text { SECUENCIAL } \\
\text { - VISUAL }\end{array}$ & $\begin{array}{l}\text { Capacidad de síntesis } \\
\text { y niveles de } \\
\text { organización de } \\
\text { información. } \\
\text { Coherencia e ilación } \\
\text { de ideas. } \\
\text { Aplicación de rubrica } \\
\text { de evaluación en } \\
\text { niveles }\end{array}$ \\
\hline
\end{tabular}


Bio - grafía. Escritos sobre la Biología y su Enseñanza. ISSN 2027-1034

Edición Extraordinaria. p.p. 420- 430

Memorias del IX Encuentro Nacional de Experiencias en Enseñanza de la Biología y la Educación Ambiental. IV Congreso Nacional de Investigación en Enseñanza de la Biología.

\begin{tabular}{|c|c|c|c|c|}
\hline & & $\begin{array}{l}\text { debe analizar y } \\
\text { semaforizar. }\end{array}$ & & categorización. \\
\hline 3 & AUDIO RAE & $\begin{array}{l}\text { Posterior a lecturas, } \\
\text { se debe sintetizar a } \\
\text { información, } \\
\text { concretar y } \\
\text { categorizar yosteriormente } \\
\text { poste } \\
\text { comunicar a través } \\
\text { de medio verbal }\end{array}$ & $\begin{array}{c}\text { REFLEXIVO } \\
\text { - AUDITIVO - } \\
\text { VERBAL }\end{array}$ & $\begin{array}{l}\text { Niveles de reflexión } \\
\text { alcanzados y } \\
\text { expuestos en el audio. } \\
\text { Capacidad } \\
\text { resumen y captura de } \\
\text { esencia del autor. } \\
\text { Conexiones lógicas } \\
\text { entre información. } \\
\text { llación y coherencia } \\
\text { textual. }\end{array}$ \\
\hline
\end{tabular}

La tabla 2, muestra algunas estrategias que son aplicadas al grupo en general, pero que desde la planeación que realiza el docente reflexivo, involucran una visión diversa y diferente del aula y sus integrantes. Algunas de estas estrategias apoyaron mejor el proceso de los estudiantes en quienes se ha identificado esas tendencias, pero aun así los integrantes del grupo que presentan tendencias moderadas pueden adaptarse y triunfar en ellas. La planeación y estrategia logra visibilizar el pensamiento y el alcance de los estudiantes en relación a la temática propuesta.

Estas transformaciones implicaron en los profesores del curso la planeación desde la $\mathrm{EpC}$, con un hilo conductor relacionado con el interrogante, ¿El conocimiento profesional del profesor de ciencias se visibiliza desde sus componentes?, y el establecimiento de metas de comprensión de contenido, de método, de praxis y de comunicación, en esta última, a manera de ejemplo, se planeó que el estudiante comunicara oralmente y por escrito el proceso de planeación y grabación de una sesión de clase de ciencias, desde los resultados que se obtienen en su práctica profesional.

Ahora bien, desde los estudiantes - profesores en ejercicio, fue posible identificar tres grandes transformaciones: Primero, el reconocimiento de parte del estudiante en formación de elementos propios en relación a su tendencia de estilo de aprendizaje, que al interiorizarlos y hacerlos evidentes, se incorporan como parte integral de su potencial, encontrando en el espacio de aprendizaje oportunidades de comunicación, interacción, aprendizaje individual, socialización de conocimientos y controversia argumentada. Segundo, la diversificación de las estrategias de aprendizaje aplicadas en el contexto de aula, las cuales, se desarrollan con intencionalidades directas, buscando una mayor participación, inclusión y puesta en escena de las habilidades y cualidades de cada estudiante - profesor. En este sentido el profesor del curso conoce de antemano cuáles son los perfiles de tendencia de sus estudiantes que favorece la participación y la 
Bio - grafía. Escritos sobre la Biología y su Enseñanza. ISSN 2027-1034

Edición Extraordinaria. p.p. 420- 430

Memorias del IX Encuentro Nacional de Experiencias en Enseñanza de la Biología y la

Educación Ambiental. IV Congreso Nacional de Investigación en Enseñanza de la Biología.

visibilización. Tercero, se encuentra la fortaleza en relación al aprendizaje colaborativo, la inclusión de roles de trabajo, la individualización de los procesos de participación y de evaluación en ciencias soportada en rúbricas, para establecer criterios claros y objetivos, de construcción individual y colectiva y de aplicación en momentos de autoevaluación, coevaluación y heteroevaluación. El estudiante - profesor en formación es partícipe de las estrategias que debe aplicar en su aula de ejercicio y ello lo conduce a encontrar fortalezas, debilidades, aciertos o campos nuevos para sus estudiantes.

Tabla 3. Análisis de Pearson entre las categorías establecidas para los estilos de aprendizaje.

\begin{tabular}{|c|c|c|c|c|c|c|c|c|c|}
\hline & \multicolumn{8}{|c|}{ DISTRIBUCION PORCENTUAL EN EL GRUPO DE ESTUDIO N = 26} & \\
\hline \multirow{3}{*}{ AGRUPACION GENERAL POR CATEGORIAS. } & 16 & 10 & 14 & 12 & 16 & 10 & 17 & 9 & \\
\hline & ACTIVO & REFLEXIVO & SENSITIVO & INTUITIVO & VISUAL & VERBAL & SECUENCIAL & GLOBAL & \\
\hline & 61,5 & 38,5 & 53,8 & 46,2 & 61,5 & 38,5 & 65,4 & 34,6 & CORRELACION DE PEARSON \\
\hline \multirow{4}{*}{ AGRUPACION PORCENTUAL EN MUJERES } & & DISTR & BUCION POR & EENTUAL EN & GRUPOD & UJERES $\mathrm{N}$ & $=10$ & & \multirow{4}{*}{0,793725393} \\
\hline & 7 & 4 & 5 & 6 & 7 & 4 & 6 & 5 & \\
\hline & ACTIVO & REFLEXIVO & SENSITIVO & INTUITIVO & VISUAL & VERBAL & SECUENCIAL & GLOBAL & \\
\hline & 63,6 & 36,4 & 45,5 & 54,5 & 63,6 & 36,4 & 54,5 & 45,5 & \\
\hline \multirow{4}{*}{ AGRUPACION PORCENTUAL EN HOMBRES } & & DISTR & BUCION POR & ENTUAL EN & GRUPO DE & MBRES $N$ & $=16$ & & \multirow{4}{*}{0,95006925} \\
\hline & 9 & 6 & 9 & 6 & 9 & 6 & 11 & 4 & \\
\hline & ACTIVO & REFLEXIVO & SENSITIVO & INTUITIVO & VISUAL & VERBAL & SECUENCIAL & GLOBAL & \\
\hline & 60,0 & 40,0 & 60,0 & 40,0 & 60,0 & 40,0 & 73,3 & 26,7 & \\
\hline
\end{tabular}

El análisis de correlación de Pearson, según Sampieri (Hernandez, 2010) es una prueba estadística para analizar la relación entre dos variables medidas en un nivel por intervalos o de razón. En esa línea, se relacionaron las puntuaciones obtenidas en todo el grupo para las cuatro dimensiones y sus ocho categorías con las puntuaciones de agrupación por género. Los valores de 0,793725393 para el grupo de mujeres de la muestra, indica una correlación alta entre la distribución de tendencias en el estilo de aprendizaje de toda la muestra y el grupo de mujeres. De igual manera el valor de 0,95006925 para el grupo de hombres indica una alta correlación en la validez y aplicación de la prueba. Además, a nivel grupal las variables de género difieren, sin ser un elemento determinante en la implementación de las estrategias o el desarrollo de la prueba y por tanto los resultados admiten la distribución por géneros.

En relación a estos casos de correlación entre tendencias, los profesores del curso desarrollaron ejercicios integradores en el aula, con planeaciones bajo el marco de la EpC y desde allí se implementan acciones que se convierte en sí, en un ejercicio de reflexión sobre la práctica pedagógica, la predominancia o ausencia de alguno de estos elementos y las relaciones que se presentan en sus dinámicas y contextos de aula. 
Bio - grafía. Escritos sobre la Biología y su Enseñanza. ISSN 2027-1034

Edición Extraordinaria. p.p. 420- 430

Memorias del IX Encuentro Nacional de Experiencias en Enseñanza de la Biología y la

Educación Ambiental. IV Congreso Nacional de Investigación en Enseñanza de la Biología.

\section{CONCLUSIONES}

- Se reconoce en el grupo de estudiantes - profesores en formación la caracterización del contexto de los estudiantes, permitiendo una mirada de cada uno de ellos. Los profesores en formación presentan tendencias en los estilos de aprendizaje visual y secuencial, lo que permitió transformar la práctica pedagógica de formación, desde la implementación de estrategias de Enseñanza para la Comprensión.

- Identificar esas tendencias en los estudiantes es una herramienta de caracterización que obliga a la planeación y ejecución de propuestas pedagógicas acordes, de inclusión y orientadas a la reflexión. En sí los profesores del curso planean y desarrollan estrategias de aplicación directa en sus aulas facilitando transformaciones en los procesos pedagógicos y curriculares en las instituciones en donde desempeñan sus funciones.

- El tener una relación entre los estilos de aprendizaje y las estrategias desarrolladas en el Énfasis de Docencia para el Desarrollo del Pensamiento Científico y Tecnológico, ha permitido mejorar y evidenciar los procesos de seguimiento, evaluación de los estudiantes. Se evidencia un mejor desempeño, mayor participación, interés en el desarrollo de la actividad desde la inclusión en los ejercicios de aula.

- Los estudiantes - profesores en ejercicio tienen un acercamiento al aprendizaje experiencial, vivencian sus procesos desde los estilos de aprendizaje y al reconocer las acciones suelen aplicar en sus propios contextos.

- En general las estrategias aplicadas en la investigación demuestran una integración amplia entre el estilo de aprendizaje, la intencionalidad dentro del aula y la forma de obtener y evaluar resultados en términos de aprendizaje, motivación, reflexión y propuestas de cambio desde el docente en formación, en especial cuando en sus ejercicios profesionales implementan dichas acciones y obtienen mayor motivación, inclusión y aprendizaje en sus aulas.

\section{REFERENCIAS BIBLIOGRÁFICAS}

Beltrán J.A. (2003). Estrategias de aprendizaje. Revista de Educación, P. 55 - 73.

Castaño C, G. (2004). Independencia de los estilos de aprendizaje de las variables cognitivas y afectivo emocionales. Obtenido de Universidad Complutense de Madrid. Departamento de Psicología Diferencial y del Trabajo: http://pendientedemigracion.ucm.es/BUCMtesis/psi/ucm-t28051.pdf

Cazau, P. (2004). Estilos de aprendizaje: Generalidades. Obtenido de www.gale on.com/pcazau/guia_01. 
Bio - grafía. Escritos sobre la Biología y su Enseñanza. ISSN 2027-1034

Edición Extraordinaria. p.p. 420- 430

Memorias del IX Encuentro Nacional de Experiencias en Enseñanza de la Biología y la

Educación Ambiental. IV Congreso Nacional de Investigación en Enseñanza de la Biología.

Chiang, M. T. (2016). Estilos de enseñanza y aprendizaje:¿ cómo dialogan en la práctica?. Journal of Learning Styles, 9(17). Journal of Learning Styles.

Felder, R., \& Soloman, B. (1991). Index of Learning Styles Questionnaire - North Carolina State University. Obtenido de https://www.engr.ncsu.edu/learningstyles/ilsweb.html: https://www.engr.ncsu.edu/learningstyles/lisweb.html

Felder, R. M. (1988). Estilos de aprendizaje y de enseñanza en la educación de ingeniería. [Versión electrónica]. Ing. Educación, 78(7), 674-681.

Hernández S, R. (2010). Metodología de la Investigación. México: Mc Graw Hill.

Huizar, M.V. (2009). Estilos de aprendizaje y estilos de pensamiento. Educación y Desarrollo, 11, 19-30.

Ospina, M., Salazar, L., \& Meneses, J. (2013). Modelos de estilos de aprendizaje: una actualización para su revisión y análisis. Learning Styles Models: An upgrade for their revision and analysis/Modelos de estilos de aprendizaje., 79-105.

Pantoja O, M. A., Duque S, L. I., \& Correa M, J. S. (2013). Modelos de estilos de aprendizaje: una actualización para su revisión y análisis. Revista Colombiana de Educación, 79 - 105.

Sternberg, R. J. (1999). Estilos de pensamiento. Claves para identificar nuestro modo de pensar y enriquecer nuestra capacidad de reflexión. Barcelona: Paidos.

Tavares, C. C. (2007). Caracterizando el estilo de aprendizaje de poblaciones de estudiantes heterogéneas. Encuentro, 1-9.

Valbuena, E. O. (2007). Memorias de trabajo de Grado. Madrid - España: Universidad Complutense de Madrid. 